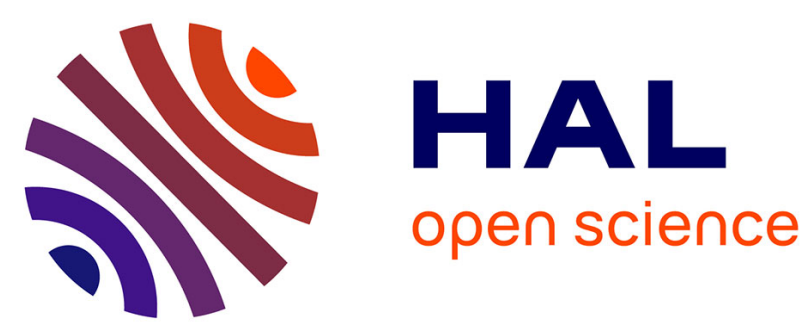

\title{
ÉVALUATION DE L'ADHÉRENCE PAR ULTRASONS HAUTE-FRÉQUENCE
}

Mohammadi Ouaftouh, Wei-Jiang Xu, Mohamed Ourak, Bertrand Nongaillard

\section{To cite this version:}

Mohammadi Ouaftouh, Wei-Jiang Xu, Mohamed Ourak, Bertrand Nongaillard. ÉVALUATION DE L'ADHÉRENCE PAR ULTRASONS HAUTE-FRÉQUENCE. Journal de Physique IV Proceedings, 1992, 02 (C1), pp.C1-835-C1-838. 10.1051/jp4:19921182 . jpa-00251144

\section{HAL Id: jpa-00251144 https://hal.science/jpa-00251144}

Submitted on 1 Jan 1992

HAL is a multi-disciplinary open access archive for the deposit and dissemination of scientific research documents, whether they are published or not. The documents may come from teaching and research institutions in France or abroad, or from public or private research centers.
L'archive ouverte pluridisciplinaire HAL, est destinée au dépôt et à la diffusion de documents scientifiques de niveau recherche, publiés ou non, émanant des établissements d'enseignement et de recherche français ou étrangers, des laboratoires publics ou privés. 


\title{
EVALUATION DE LADHERENCE PAR ULTRASONS HAUTE-FREQUENCE
}

\author{
M. OUAFTOUH, W.J. XU, M. OURAK et B. NONGAILLARD \\ Laboratoire d'Opto-Acousto-Electronique, URA 832 CNRS, Université de Valenciennes, ENSIMEV, \\ BP. 311, F-59304 Valenciennes, France
}

\begin{abstract}
Numerous studies have already been performed for estimating the adhesion between two materials using ultrasound. The results obtained, mainly with Lamb waves, don't lead to a quantitative evaluation of adhesion. We investigated the use of high frequency, 50 to $500 \mathrm{MHz}$, bulk (longitudinal and shear) ultrasonic waves for evaluating the adhesion from the reflection or transmission coefficient at an interface.
\end{abstract}

Résumé : De nombreux essais ont été réalisés afin de caractériser l'adhésion entre deux matériaux par ondes ultrasonores. Ces essais, souvent réalisés en ondes guidées ou en ondes longitudinales basses fréquences sous incidence normale ( $<15 \mathrm{MHz}$ ), permettent difficilement d'évaluer quantitativement l'adhésion. Nous nous proposons de présenter les résultats de mesures du coefficient de réflexion sur divers échantillons représentatifs de mauvais collages. Ces essais ont été effectués en ondes longitudinales et transversales pour des fréquences comprises entre 50 et $500 \mathrm{MHz}$.

\section{INTRODUCTION}

Les structures modernes font de plus en plus appel à des techniques d'assemblages ou de protection (dépôt céramique, collages structuraux, ...) où l'évaluation non destructive de la qualité d'adhérence à l'interface est primordiale pour assurer la fiabilité de l'assemblage.

Pendant longtemps, la majorité des études sur le contrôle non destructif de la qualité d'adhérence a été menée en utilisant des ondes longitudinales (jusqu'à $15 \mathrm{MHz}$ ) sous incidence normale [1-5]. Les résultats obtenus sont peu fiables et ont montré l'incapacité de cette technique à résoudre un tel problème surtout dans le cas des collages structuraux. Toutefois, quelques correlations entre les mesures ultrasonores et la qualité du collage ont été rapportées dans quelques publications. Ces résultats concernent essentiellement les défauts de délaminage à l'interface métal-adhésif ou des défauts de cohésion au sein de l'adhésif. Récemment, des études ont montré, d'une part, l'intérêt de solliciter acoustiquement le joint de colle dans le sens de sa plus faible tenue mé canique (cisaillement) [6-8], mais également l'intérêt de monter en fréquence pour quantifier l'adhésion [9]. Les ondes de plaque et les ondes transversales permettent de générer ce type de sollicitations. L'étude de la propagation des ondes guidées dans un joint collé permet d'avoir accès à l'évolution des courbes de dispersion en fonction de l'épaisseur de la couche d'adhésif et de la nature des liaisons mécaniques aux interfaces (bonne ou mauvaise adhésion). Ces liaisons se traduisent sur le plan mathématique par des conditions aux limites différentes :

les conditions parfaites, pour lesquelles il y a continuité des déplacements et des contraintes au niveau de l'interface, sont associées à un contact rigide.

les conditions imparfaites [10] consistent à écrire la continuité des contraintes normale et tangentielle et des relations de proportionnalité entre celles-ci et la différence des composantes du déplacement de part et d'autre de l'interface. Les coefficients de proportionnalité normal $\mathrm{K}_{\mathrm{n}}$ et tangentiel $\mathrm{K}_{\mathrm{t}}$ sont censés caractériser théoriquement la qualité d'adhérence au niveau de l'interface. Ces condi- 
tions, dites de sauts de déplacements, sont des conditions intermédiaires entre les conditions parfaites et les conditions glissantes correspondant à l'annulation de la composante tangentielle des contraintes.

Les résultats obtenus à l'aide de ce type d'onde [11,12] montrent que les défauts d'adhésion ne modifient pas de façon significative les courbes de dispersion des modes de plaque à cause, sans doute, de la forte rupture d'impédance entre l'adhésif et les substrats. De plus, les différences observées dépendent fortement de l'épaisseur du film de colle. Dans cet article nous présentons les résultats obtenus à l'aide des ondes de volume haute fréquence $(>50 \mathrm{MHz})$. Nous comparons, en particulier, le contrôle en ondes transversales sous incidence normale au contrôle en ondes longitudinales également sous incidence normale.

\section{ONDES LONGITUDINALES ET TRANSVERSALES HAUTE-FREQUENCE}

L'étude de la réflexion d'une onde de volume de type longitudinal ou transversal sur une interface simple aluminium-colle [13] montre tout l'intérêt de l'utilisation d'ondes transversales pour le contrôle de l'adhérence, compte tenu de la sollicitation en cisaillement de l'interface qu'elles réalisent. Elle fait apparaître également une grande sensibilité du contrôle sous incidence normale pour la détection des contacts sans adhésion. Cette étude théorique suppose que l'on puisse séparer, dans le temps, les signaux réfléchis sur chaque interface. Ceci est rendu possible en haute fréquence grâce à la faible durée du signal émis par rapport au temps de vol dans chaque couche.

Nous avons réalisé, dans un premier temps, des mesures en ondes longitudinales à $100 \mathrm{MHz}$ et en ondes transversales à $30 \mathrm{MHz}$ sur des échantillons composés de deux plaques d'aluminium collées par un film de colle de type résine époxyde. Les défauts d'adhésion ont été réalisés par pulvérisation d'un film très mince de téflon sur l'une des surfaces des deux plaques en contact avec l'adhésif. L'ensemble des résultats expérimentaux obtenus avec ce type d'ondes met en évidence des écarts tès faibles d'amplitude entre les régions avec et sans pulvérisation de téflon. Ces écarts sont de l'ordre de 2 à $3 \%$ au maximum, ce qui semble recouper des mesures effectuées par sonde laser sur des matériaux transparents (fig.1). Ces variations peuvent s'expliquer aussi bien par une mauvaise qualité d'adhérence que par la présence du film de téflon ou par les erreurs de mesures.

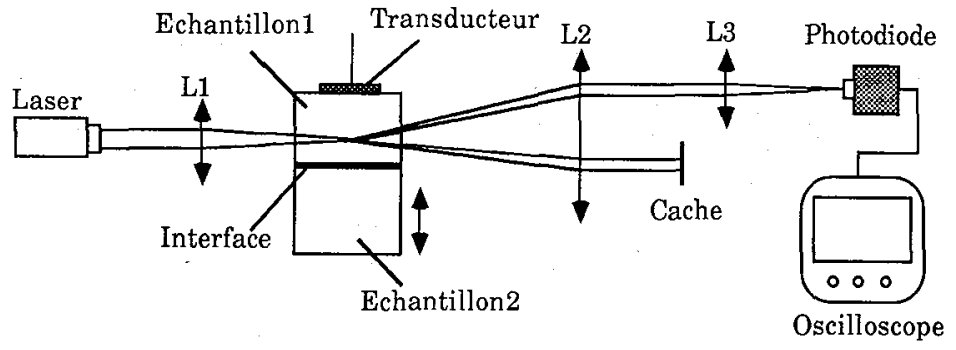

Fig.1 : Système optique pour la mesure des amplitudes des champs ultrasonores.

Ces constatations nous ont amené à travailler avec des échantillons formés de deux matériaux dont les surfaces mises en contact sont bien polies et parfaitement planes (silice-silice ou silice-corindon). Une adhérence optique est réalisée en comprimant légèrement les deux matériaux entre eux de manière à chasser l'air existant au niveau de l'interface qui les sépare. Les essais effectués à $100 \mathrm{MHz}$ en ondes longitudinales et en ondes transversales mettent en vidence une meilleure sensibilité des ondes de cisaillement vis-à-vis de la qualité d'adhérence au niveau de l'interface (fig.2). Des résultats identiques ont été également observés pour des fréquences comprises entre 100 et $500 \mathrm{MHz}$. Nous avons constaté également à partir des mesures effectuées sur les échantillons silice-corindon (fig.3), que les variations d'amplitude relatives à un contact sans adhésion sont faibles par rapport à celles causées par la rupture d'impédance entre les deux matériaux mis en contact. Ce résultat explique, sans doute, les faibles écarts obtenus sur les échantillons collés aluminium-colle-aluminium.

Afin de verifier la possibilité du contrôle de la qualité d'adhérence par les ondes transversales, nous avons effectué des mesures pour différentes fréquences (entre 50 et $500 \mathrm{MHz}$ ) sur des échantillons en silice présentant deux types de contact (fig.4): 
une mauvaise adhésion, simulée par un contact optique.

une bonne adhésion, obtenue en collant les deux échantillons de silice à l'or ou à l'indium. Ce collage est réalisé en évaporant sous vide sur chacune des surfaces à coller une couche d'or ou d'indium d'épaisseur très faible $(\approx 1500 \AA)$. De plus, une couche de chrome $(\approx 100 \AA)$ est introduite au préalable de façon à assurer l'adhérence de l'or ou de l'indium sur la silice.
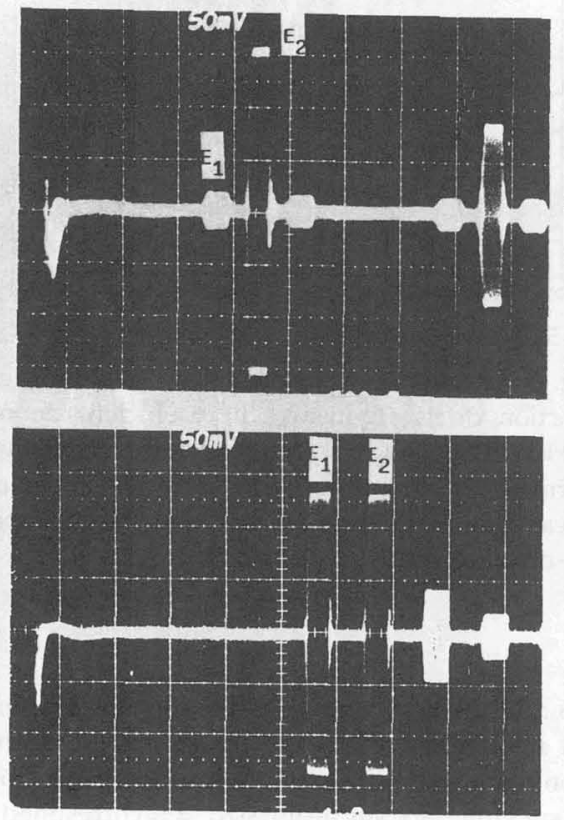

$2 . b$

Fig.2 : Diagrammes temporels des échos d'une onde longitudinale à $100 \mathrm{MHz}$ (fig.2.a) et d'une onde transversale à $100 \mathrm{MHz}$ (fig.2.b) sous incidence normale sur un échantillon silice-silice présentant une adhérence optique.

$E_{1}$ : écho d'interface $E_{2}$ : écho de fond

Les résultats obtenus sont représentés sur la figure 5. Nous pouvons constater tout d'abord que le coefficient de réflexion au niveau de l'interface croit avec la fréquence (fig.5.a), ce qui est conforme aux résultats théoriques obtenus en utilisant les conditions de sauts de déplacements au niveau de l'interface silice-silice (fig.6), quoique les valeurs théoriques soient supérieures à celles mesurées. Nous pouvons remarquer également que les ondes transversales permettent de bien distinguer les deux qualités d'adhérence vu l'écart sur le coefficient de rêflexion correspondant aux deux types de contacts (fig.5.b).

\section{CONCLUSION}

L'utilisation des ondes ultrasonores haute fréquence nous a permis de confirmer la faible sensibilité des ondes longitudinales vis-à-vis des défauts d'adhésion. Par contre, nous avons montré que les ondes
Fig. 3 : Diagrammes temporels des échos d'une onde longitudinale à $100 \mathrm{MHz}$ (fig.3.a) et d'une onde transversale à $100 \mathrm{MHz}$ (fig.3.b) sous incidence normale sur un échantillon corindon-silice présentant une adhérence optique.

$$
\mathrm{E}_{1} \text { : écho d'interface } \mathrm{E}_{2} \text { : écho de fond }
$$

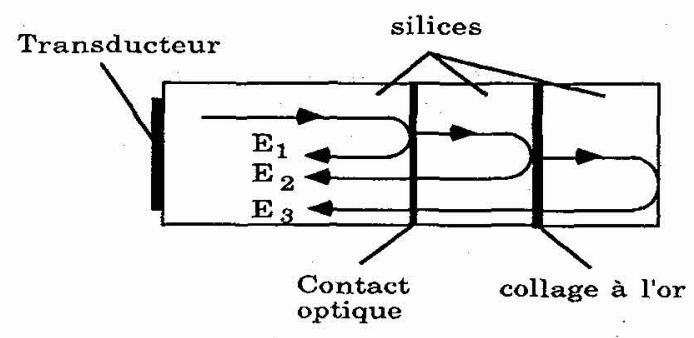

Fig.4 : Géométrie des échantillions utilisés. 
transversales sont bien adaptées à la détection de ce type de défauts. Du point de vue théorique, une amélioration de la modélisation reste à effectuer en considérant, par exemple, des relations non linéaires entre les contraintes et les sauts de déplacements au niveau de l'interface.
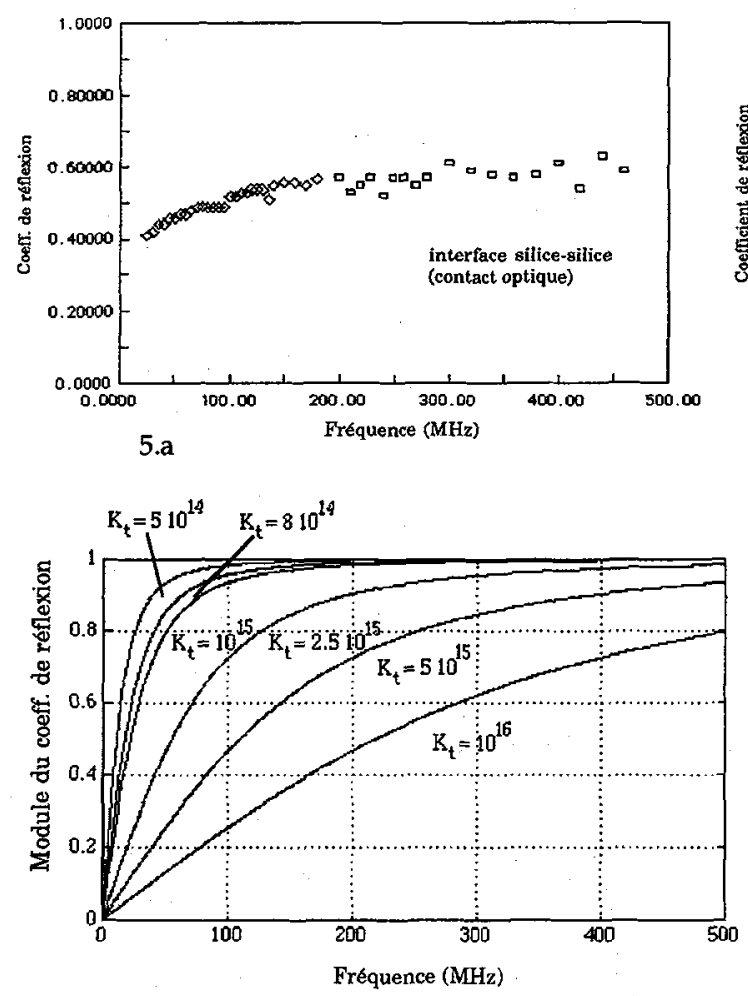

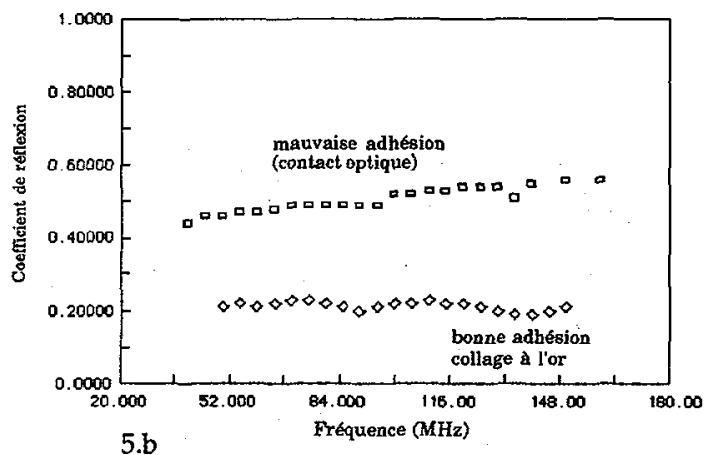

Fig. 5 : Résultats expérimentaux : évolution en fonction de la fréquence du coefficient de réflexion d'une onde transversale sous incidence normale sur linterface silice-silice (fig.5.a : dans le cas d'une mauvaise adhésion; fig.5.b : comparaison entre les deux qualités d'adhérence).

Fig. 6 : Résultats théoriques : évolution en fonction de la fréquence du module du coefficient de réflexion d'une onde transversale sous incidence normale sur l'interface silice-silice $\left(K_{t}=\infty\right.$ correspond à un contact parfait et $K_{t}=0$ à contact glissant).

\section{Remerciements}

Nous tenons à remercier le Laboratoire Central de recherche de l'Aérospatiale (Suresnes - France) et la DRET pour leurs aide et collaboration.

\section{REFERENCES}

[1] Segal, E. and Rose,J.L. Research Techniques in NDT (Academic Press, 1980), 4, Chap.8.

[2] Rose,J.L. and Mayer P.A. Mater. Eval. (1973), 31, 6, 109-114.

[3] Chang, F.H., Flynn, P.L., Gordon, D.E., and Bell,J.R. IEEE Trans. Sonics Ultrason. (1976), SU-23, 5, 334-338.

[4] Rose, J.L., Avioli, M.J.Jr, and Bilgram,R. Brit. J. NDT (1983), 24, 2, 67-71.

[5] Alers, G.A., Flynn, P.L., and Buckley,M.J. Mater. Eval. (1977), 35, 77-84.

[6] Rokhlin, S.I., and Marom, D. J. Acoust. Soc. Am. (1986), 80, 2, 245-258.

[7] Pilarski, A., and Rose, J.L. NDT Internal. (1988), 21, 4, 241-246.

[8] Pilarski, A., Mater. Eval. (1985), 43, 6, 765-770.

[9] Nestleroth, J.B., Rose,J.L., Lecuru, D., and Budillon, E., Rev. Prog. Quant. NDE (Plenum Press, 1987), 6B, 1787-1795.

[10] Schoenberg, M. J. Acoust. Soc. Am. (1980), 68, 5, 1516-1521.

[11] Léomy, F., de Billy, M., Quentin, G., Benelmostafa, Y., de Belleval, J.F., Mercier, N., Molinero, I., and Lecu$\mathrm{ru}$, D., Review of Progress in Q.N.D.E. (Brunswick), 9, 1989.

[12] Ouaftouh, M., Ourak, M., Xu, W.J., Nongaillard, B., Lefebvre, J.E., and Rouvaen, J.M. Ultrason. Internat'l Symp., Le Touquet, France, July 1991 (Proceedings to be published).

[13] Ouaftouh, M., Lefebvre, J.E., Nongaillard, B., Rouvaen, J.M. and Joseph, S. 4th Inter. Conf. Qual. in Electr. Comp.. (April 1989), Bordeaux (France). 